Kosten und Nutzen eines Nationalparks Donau-Auen

\section{Wie teuer ist uns die Natur?}

\section{Die verschiedenen Varianten einer Nationalparkplanung Donau-Auen umwelt- ökonomisch zu beurteilen, war die Augabe des Instituts für Finanzwissenschaft und Infrastrukfurpolitik an der Technischen Universität Wien. Welche Bedeu- tung dabei insbesondere einer ökologisch sensiblen Kosten-Nutzen-Analyse zukommt, zeigt der folgende Beitrag.}

Von Michael Kosz

$\mathrm{D}$ ie Ökonomie bietet zur Beantwortung der Fragestellung: „Wie teuer ist uns die Natur?" eine Reihe von (mehr oder weniger verläßlichen) Methoden an. Grundsätzlich wird davon ausgegangen, daß diese Meßverfahren bereits vorhandene Wertschätzungen für Umweltgüter transparent machen (können). Unterschieden wird in indirekte und direkte Bewertungsmethoden: Unter die indirekten Methoden fallen z.B. die Messung von Defensivkosten zur Abwehr oder Reparatur einer Umweltbeeinträchtigung, der Reisekostenansatz oder die ökonomische Analyse von Mietpreisunterschieden in Abhängigkeit von der Luftqualität oder den Lärmimmissionen. Indirekte Methoden fußen also auf beobachtbaren Markttransaktionen (und -preisen), die durch unterschiedliche Wertschätzungen für Umweltgüter

Barwerte der volkswirtschafflichen Kosten-Nutzen-Saldi (in Mio. Schilling)

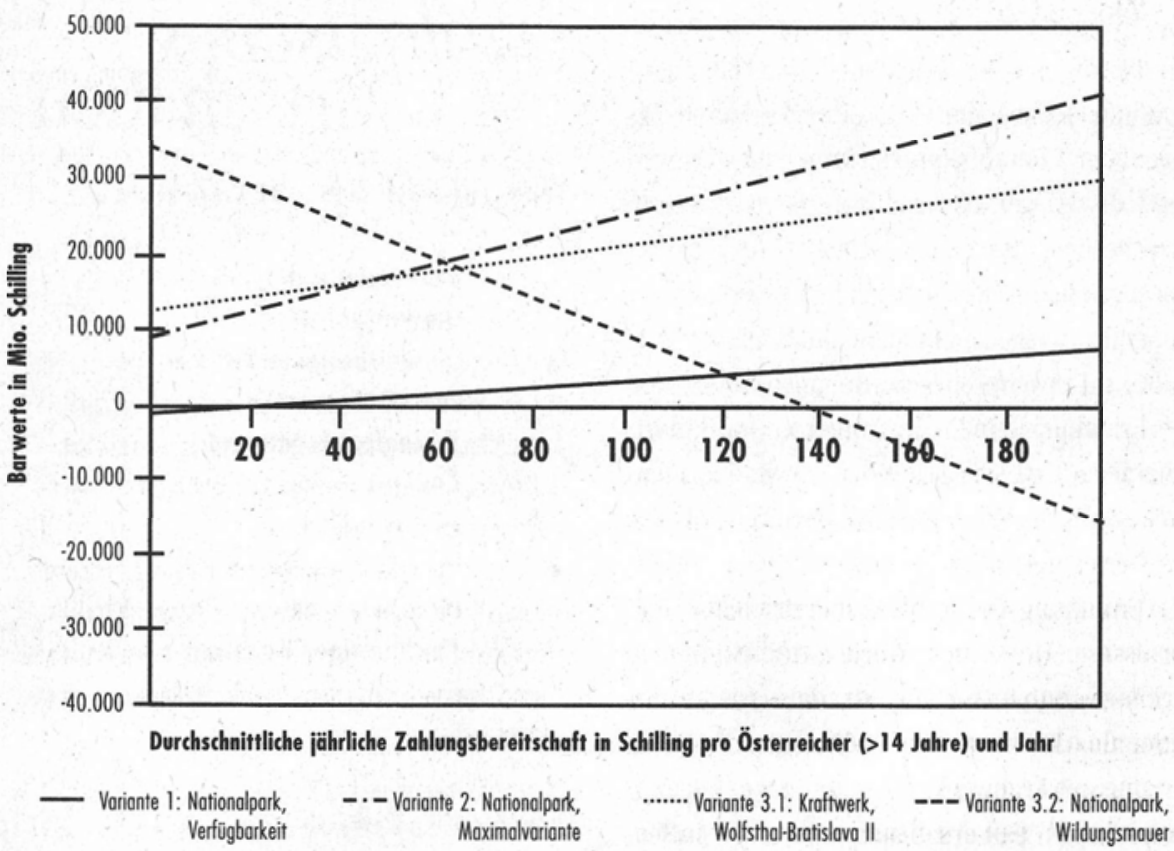

Quelle: Schönbōck et. al., 1996
Auen liegt zwischen den Hauptstädten Bratislava und Wien entlang der österreichischen Donau (Länge rund 50 Kilometer, Breite der DonauAuen maximal fünf Kilometer).

\section{Zu untersuchende Varianten}

In der vorliegenden Kosten-Nutzen-Analyse werden der absolute volkswirtschaftliche Wohlfahrtseffekt (Barwert der jährlichen Kosten-Nutzen-Saldi) und die relative volkswirtschaftliche Rentabilität (interner volkswirtschaftlicher Zinssatz des eingesetzten Kapitals) ausgewählter Varianten der Gestaltung des österreichischen Donauabschnittes östlich von Wien und dessen voraussichtliche Nutzung in der Periode 1993 bis 2068 untersucht. In Variante 1 (Nationalpark - Verfügbarkeit) wird ein Nationalpark auf jenen Flächen, die sich in öffentlichem Besitz befinden, errichtet (Fläche des Nationalparks: 9.300 Hektar). Es werden keine flußbaulichen Maßnahmen gesetzt, die der Erosion der Donausohle, welche durch die Donauregulierung Ende des vorigen Jahrhunderts verursacht wurde und langfristig durch die Absenkung des Grundwasserspiegels infolge niedrigerer Wasserspiegellagen des Hauptstromes ein ernsthaftes ökologisches Problem darstellen kann, entgegenwirken. Diese Variante hätte sehr gute Chancen auf internationale Anerkennung als Nationalpark gemäß den International Union for the Conservation of Nature and Naturel Resources (IUCN)-Kriterien.

In Variante 2 (Nationalpark - Maximalvariante) wird ein Nationalpark auch auf Flächen, die sich in privatem Besitz befinden, errichtet (gesamte Fläche 11.500 Hektar). Umfangreiche flußbauliche Maßnahmen, vor allem die Sohlerollierung (Sohlepflasterung), sollen die weitere Eintiefung der Donau verhindern. Es soll durch die Niederwasserregulierung eine durchgängige Fahrwassertiefe von 270 Zentimeter erreicht werden, um die Schiffahrtsverhältnissse zu verbessern. Diese Variante hätte ausgezeichnete Chancen auf internationale Anerkennung.

In den Varianten 3.1 (Kraftwerk Wolfsthal-Bratislava II) und 3.2 (Kraftwerk Wildungsmauer) wird jeweils ein Stauwerk, das der Stromerzeugung und der Schiffahrt dient, errichtet. Flußbauliche Maßnahmen, wie die Stabilisierung der Donausohle durch Sohlerollierung und die Niederwasserregulierung, sind auch in diesen Varianten notwendig. Die Varianten mit Kraftwerk haben verringerte (3.1) beziehungsweise kaum (3.2) Chancen auf internationale Anerkennung, 
würden jedoch je nach Variante zwischen 1,5 bis 2,7 Prozent des österreichischen Strombedarfs produzieren und zu einer Einsparung von fossilen Brennstoffen und damit verbundenen $\mathrm{CO2}$ Emissionen führen.

\section{- Ergebnisse der Untersuchung}

Aus den Problemdimensionen (vgl. Kasten) wurden ökonomisch bewertbare Hauptvariablen gebildet, die in die Kosten-Nutzen-Analyse Eingang fanden. Im folgenden werden aus den Hauptvariablen die aus umweltökonomischer Sicht interessantesten herausgegriffen.

Die Reisekostenmethode ergab den Reiseaufwand, den BesucherInnen auf sich nehmen, um das Nationalparkgebiet zu besuchen. Grundüberlegung dabei ist, daß die Reise dann nicht getätigt worden wäre, wenn der Erholungswert geringer als der Reiseaufwand, um in das Gebiet zu gelangen, gewesen wäre. Festgestellt wurde, daß pro Person ein durchschnittlicher Reiseaufwand von rund 18 österreichischen Schilling pro Besuch entsteht (dies ergibt einen insgesamten Erlebniswert des Nationalparkgebietes von rund 38,5 Millionen Schilling pro Jahr). Werden nicht nur die Reiseaufwendungen, sondern der gesamte mit dem Besuch verbundene Aufwand herangezogen, so ergibt sich ein ProKopf-Aufwand von fast 165 Schilling pro Besuch (gesamter Erlebniswert des Nationalparkgebietes: 181,1 Millionen Schilling pro Jahr).

Den beiden Methoden ist gemeinsam, daß die Abgrenzungen zwischen jenem Aufwand, der nationalparkspezifisch ist, und jenem, der auch ohne den Nationalparkbesuch getätigt worden wäre, sehr problematisch ist. Eine österreichweit durchge-führte Repräsentativumfrage nach der Zahlungsbereitschaft für einen Eintrittspreis ergab einen fiktiven (hypothetischen) Wert (1) von rund 80 Schilling pro Person und Besuch. Dieser Betrag wurde in weiterer Folge für die Gesamtanalyse herangezogen, da er jene Zahlungsbereitschaft darstellt, die direkt mit dem Erholungs- und Freizeitsnutzen zusammenhängt und von den Befragten die einzelnen Varianten (,reiner“ Nationalpark; Varianten mit Kraftwerk) auch unterschiedlich eingeschätzt wurden.

Zur Messung des Existenz-, Options- und Vermächtniswertes des Nationalparks Donau-Auen (Erhalt der Augebiete) wurde eine repräsentative, österreichweite Zahlungsbereitschaftsbefragung durchgeführt. Für die größte Nationalparkvariante ergab sich eine Zahlungsbereitschaft (pro Kopf und Jahr) von rund 920 Schilling bei Einbeziehung nur jener Befragten, die eine Zahlungsbereitschaft geäußert haben. Bei Durchschnittsbildung über alle Befragten (also auch jener, die keine Zahlungsbereitschaft geäußert haben) ergibt sich ein jährlicher Betrag von rund 330 Schilling. Am wichtigsten ist den Befragten der Existenzwert (Wert der Natur an sich) mit 50 Prozent der Zahlungsbereitschaft, gefolgt vom Vermächtniswert (Erhalt der Natur für zukünftige Generationen, 37 Prozent) und dem 0ptionswert (Erhalt der Natur für zukünftige Nutzung durch die Befragten selbst, 13 Prozent). Bei Annahme einer unendlich langen Planungsperiode beträgt der Gegenwartswert des Nationalparks unabhängig von menschlicher Nutzung (total economic value) bei einem Zinssatz von zwei Prozent pro Jahr rund 110 Milliarden Schilling.

Die durchgeführte Kontingenzbefragung zeigte, daß die Zahlungsbereitschaft neben anderen Faktoren signifikant vom Einkommen der Befragten abhängt. Je höher das Einkommen, desto höher auch die geäußerte Zahlungsbereitschaft. Dies verdeutlicht ein wesentliches Problem der Kosten-Nutzen-Analyse, welche Verteilungsprobleme konzeptionell außer Acht läßt, obwohl die Verteilungssituation in einer Gesellschaft selbst ein öffentliches Gut darstellen kann. Auf Verteilungsprobleme konnte jedoch in der vorliegenden Kosten-Nutzen-Analyse nicht eingegangen werden, was einen Schwachpunkt darstellt.

\section{Was ist effizient?}

Bei der Frage: „Wie teuer ist uns die Natur?“ ist nicht nur die absolute Größe der Wertschätzung gegenüber Umweltgütern zu beachten, sondern auch der Vergleich zu allen anderen Gütern (privaten wie öffentlichen) herzustellen (2). Bei der Ergebnisermittlung, welche Variante nun die aus volkswirtschaftlicher Sicht effizienteste sei, wurde ein Zweistufenverfahren angewandt:

(1) Ermittlung der Ergebnisvariablen volkswirtschaftlicher Barwert, interne Verzinsung und Barwertrate je Variante ohne Berücksichtigung des Wertes der Natur.

(2) Ermittlung des Zahlungsbereitschaftserfordernisses (Höhe des Wertes der Natur) je Ergebnisvariable, um die Maximalvariante des Nationalparks besser zu stellen als die beste Variante mit Kraftwerk.

Im konkreten Fall ergab sich, daß unter Außerachtlassung der Beeinträchtigung der Augebiete durch ein Laufkraftwerk bei Wildungsmauer eine Lösung mit Kraftwerk volkswirtschaftlich den größten absoluten Vermögenszuwachs erbringt (die größte relative volkswirtschaftliche Rentabilität, das ist die Verzinsung des eingesetzten Kapitals und die Barwertrate, weist jedoch die Nationalpark-Maximalvariante (Variable 2) ohne Kraftwerk auf). Ist nun die Wertschätzung für einen unberührten Nationalpark so groß, daß die volkswirtschaftlichen Nutzeffekte eines Laufkraftwerkes, gemessen durch den Barwert, zumindest aufgewogen werden?

\section{Problemdimensionen zur Untersuchung der Projektvarianten:}

- Art und Umfang der wosserbaulichen Maßnahmen (hydrologische Auswirkungen) insbesondere im Hinblick ouf die negativen Effekte der im vorigen Jahrhundert durchgeführten Donauregulierung (Sohleintiefung) sowie auf die Vernetzung der strombegleitenden Augewässer und den Abbau der "harten" Uferverbouung.

- Auswirkungen der einzelnen Varianten eines Nationalparks Donau-Auen auf die Biodiversität (Vielfalt innerhalb und zwischen den Arten sowie der Ökosysteme sowie ökologische Wertigkeit der verbleibenden an freier Fliefsstrecke liegenden Auflächen).

- Energetische Nutzung (Kosten und Nutzen der Errichtung von Laufkraftwerken an der Donau) im Vergleich zu kolorischen Kraftwerken, sowie alternativ zur Einsparung von Elektrizität sowie zusötzlicher Stromimporte.

- Schutz (allenfalls Beeinträchtigung) der derzeitigen und zukünffig möglichen Trinkwassergewinnung, einerseits durch die Errichtung von Stouwerken, anderseits durch Außernutzungstellung möglicher Trinkwosserentnahmen im Falle der Einrichtung eines Nationalparks.

- Jagd, forst, fischerei- und landwirtschaftliche Nutzung bei Errichtung eines Nationalparks und Vereinbarkeit dieser Nutzung mit den strengen Nationalpark-Kriterien gemöB IUCN (allenfalls Außernutzungstellung der derzeitigen Produktion).

- Sicherung und Verbesserung der Schiffahrtsbedingungen und Perspektiven der Donau als internationaler Schiffahrtsweg (die Schiffahrt stellt sich innerhalb gewisser Grenzen als volkswirtschaftlich kostengünstige Transportmöglichkeit dar).

- Rechtslage, Akzeptanz, internationale Anerkennung eines Nationalparks Donau-Auen (Österreich ist Mitglied der IUCN und verpflichtete sich, einen Nationat park nur nach den internationalen Kriterien zu errichten).

- Erholungs- und Freizeitnutzen der verschiedenen Projektvarianten für Besucherlnnen. 
Bereits bei Heranziehung von rund 20 Prozent der erfragten Zahlungsbereitschaft wird der absolute wirtschaftliche Vorteil eines Laufkraftwerkes durch die Wertschätzungen für die Natur aufgewogen, das heißt daß bei einer Zahlungsbereitschaft von 64 Schilling pro Jahr und ÖsterreicherIn (über 14 Jahre) der Barwert der Variante 2 (Nationalpark-Maximalvariante) größer ist als der Barwert der „,besten“ Variante mit Kraftwerk.

Dies zeigt auch die Abbildung 1, in der die Barwerte der volkswirtschaftlichen Kosten-NutzenSaldi der verschiedenen Gestaltungsvarianten des österreichischen Donau-Abschnitts dargestellt sind. Die Barwerte wurden in Abhängigkeit von der mittleren jährlichen Zahlungsbereitschaft (in Schilling) der Österreicher über 14 Jahre für Augebiete errechnet. Dabei wurde ein realer Diskontierungszinssatz von zwei Prozent pro Jahr (von 1993 bis 2086) zugrunde gelegt.

Es zeigt sich, daß je höher die Zahlungsbereitschaft für einen Nationalpark ist, desto größer ist der Barwert des Nationalparks und desto geringer jener der größten Variante mit Kraftwerk (mit steigender Zahlungsbereitschaft für Augebiete entlang freier Fließstrecke nehmen die geldmäßig bewerteten Verluste durch ein Lauftkraftwerk $\mathrm{zu}$; damit sinkt der Barwert der Variante 3.2).

\section{Die Bedeutung der Kosten-Nutzen-Analyse}

Zusammenfassend läßt sich festhalten, daß die Kosten-Nutzen-Analyse (KNA) auswählter Varianten eines Nationalparks Donau-Auen folgende Zielsetzungen erfïllte:

- Die KNA diente zur Informationsbeschaffung für die EntscheidunsträgerInnen und Betroffenen, da erstmals aus wirtschaftswissenschaftlicher Sicht die einzelnen Problemdimensionen in ihrer volkswirtschaftlichen Bedeutung beurteilt werden konnten.

- Die KNA machte weiter jene kritischen Projektbestandteile transparent, die je nach Ausprägung zu gänzlich unterschiedlichen Ergebnissen führte.

- Die Messung der volkswirtschaftlichen Effizienz eines Projektes mittels der KNA mag ein abstrakter Wunsch der Ökonomie als Wissenschaft sein, jedoch sagt der volkswirtschaftliche, Vermögenszuwachs aufgrund der Durchführung eines Projektes noch sehr wenig über die Erwünschtheit eines Projektes bei einer Vielzahl weitergehender Anforderungen (Verteilungsprobleme, Akzeptanz der Anrainergemeinden usw.) aus.

- Eine ökologisch sensible und auf erfragte Zahlungsbereitschaften zurückgreifende KNA kann sich im Zusammenhang mit der Bewertung öffentlicher Projekte als umweltpolitisches Instrument erweisen, da die Präferenzen der Bevölkerung für den Erhalt oder die Verbesserung von Naturgütern auch geldmäßiges Gewicht erhält.

- Das Instrument der Zahlungsbereitschaftsbefragung selbst ist jedoch kaum allgemein vermittelbar, und die Frage, was geäußerte Zahlungsbereitschaften wirklich aussagen, größtenteils noch immer ungelöst.

\section{Anmerkungen}

1) Da aufgrund der röumlichen Struktur des Gebietes und der Vielzahl an Eintrittsmöglichkeiten kein Eintrittspreis erhoben wird und ouch politisch ein Eintrittspreis nicht durchsetzbar und wünschbar ist, handelt es sich lediglich um die Zohlungsbereitschaft für einen hypothetischen Eintrittspreis.
2) Dies ist jedenfalls ein wichtiges Ziel der Kosten-NutzenAnalyse. An dieser Stelle kann jedoch nicht darauf eingegangen werden, ob die zugrundeliegende Annahme der Substituierbarkeit zwischen Naturgütern und "man-made capitol" tatsächlich im unterstellten Umfang gegeben ist, und inwiefern die Kosten-Nutzen-Analyse überhaupt ein geeignetes Instrument zur Beurteilung öffentlicher Projekte darstellt.

\section{Literafur}

- M. Kosz: Valuing Riverside Wetlands: The Case of the "Donau-Auen" National Park. Ecological Economics 16 (2) 1996, 109-127.

- W. Schönbäck., M. Kosz, T. Madreiter, D. Gfall, B. Brezino: Kosten-Nutzen-Analyse ausgewählter Vorianten eines Nationalparks Donau-Auen. Springer Verlag, Wien - New York (erscheint im Herbst 1996).

\section{Der Autor}

Dr. Michael Kosz ist wissenschaftlicher Mitarbeiter am Instifut für Finanzwissenschaft und Infrastrukturpolitik

Kontakt: Technische Universität Wien, 1040 Wien, Karlsgasse 11, Tel. $(++43)$ (1) $58801-4321$, Fax 50427 38, E-Mail:mkosz@email.tuwien.ac.at

\section{Von der City-Logistik zur raumbezogenen Stadtlogistik}

\section{City-Logistik ist das akfuelle Schlagwort im städtischen Güterverkehr. Damit werden große Hoffnungen hinsichtlich Transportoptimierung (öberwiegend) und Umweltentlastung (teilweise) verbunden. Seit einigen Jahren werden darunter verstärkt organisatorische Innovationen für eine Neugestalfung der städtischen Warentransporte diskutiert und praktisch erprobt - so auch in den hier vorge- stellten Modellvorhaben.}

$\mathrm{E}$ Von Markus Hesse und Silke Kruspe nde 1995 wurde bereits in rund 80 deutschen Städten über den städtischen Güterund Lieferverkehr diskutiert und in 19 Städten sind nach Angaben des Bundesverbandes Logistik (BVL) e.V. bereits konkrete Aktivitäten und Projekte in Gang gesetzt worden. Diese Aktivitäten bestehen überwiegend aus Kooperationen und Pools zur Belieferung von Problemkunden und -zonen durch Speditionen (vgl. Logistik Heute 1995). Ausgehend von einigen Anfangserfolgen in der Initiierung und Erprobung von
Speditionskooperationen - teilweise rein privat, teilweise unter Beteiligung der öffentlichen Hand - wurden mit dem Schlagwort der „CityLogistik“ hohe Erwartungen geweckt. Doch konnte die praktische Umsetzung dieser Modelle vor dem Hintergrund verschiedener, zum Teil nicht deckungsgleicher Ziele, diese bisher noch nicht erfüllen. Die Erfahrungen zeigen zwar, daß entgegen anfänglicher Erwartungen Kooperation auch im Wettbewerb möglich ist. Aus Sicht der Planung mangelt es jedoch an einem überzeugenden Entwurf für eine funktionstïch- 
(c) 20I0 Authors; licensee IÖW and oekom verlag. This is an article distributed under the terms of the Creative Commons Attribution Non-Commercial No Derivates License (http://creativecommons.org/licenses/by-nc-nd/3.o/), which permits unrestricted use, distribution, and reproduction in any medium, provided the original work is properly cited. 